\title{
DETERMINATION OF THE ELECTRON CYCLOTRON CURRENT DRIVE PROFILE
}

by
T.C. LUCE, C.C. PETTY, D.I. SCHUSTER* and M.A. MAKOWSKI

This is a preprint of a paper presented at the Eleventh Joint Workshop on Electron Cyclotron Emission and Electron Cyclotron Resonance Heating, October 4-8, 1999 in Oharai, Ibaraki, Japan and to be published in The Proceedings.

*Brown University, Providence, Rhode Island.

tLawrence Livermore National Laboratory, Livermore, California.

Work supported by

the U.S. Department of Energy

under Contract Nos. DE-AC03-99ER54463 and W-7405-ENG-48

GA PROJECT 30033

NOVEMBER 1999 


\section{DISCLAIMER}

This report was prepared as an account of work sponsored by an agency of the United States Government. Neither the United States Government nor any agency thereof, nor any of their employees, make any warranty, express or implied, or assumes any legal liability or responsibility for the accuracy, completeness, or usefulness of any information, apparatus, product, or process disclosed, or represents that its use would not infringe privately owned rights. Reference herein to any specific commercial product, process, or service by trade name, trademark, manufacturer, or otherwise does not necessarily constitute or imply its endorsement, recommendation, or favoring by the United States Government or any agency thereof. The views and opinions of authors expressed herein do not necessarily state or reflect those of the United States Government or any agency thereof. 


\section{DISCLAIMER}

Portions of this document may be illegible in electronic image products. Images are produced from the best available original document. 


\title{
Determination of the electron cyclotron current drive profile
}

\author{
T.C. Luce, C.C. Petty, D.I. Schuster, ${ }^{*}$ M.A. Makowski ${ }^{\dagger}$ \\ General Atomics, P.O. Box 85608, San Diego, California 92186-5608 \\ *Brown University, Providence, Rhode Island. \\ † Lawrence Livermore National Laboratory, Livermore, California.
}

\begin{abstract}
Evaluation of the profile of non-inductive current density driven by absorption of electron cyclotron waves (ECCD) using time evolution of the poloidal flux indicated a broader profile than predicted by theory. To determine the nature of this broadening, a 1-1/2 $D$ transport calculation of current density evolution was used to generate the signals which the DIII-D motional Stark effect (MSE) diagnostic would measure in the event that the current density evolution followed the neoclassical Ohm's law with the theoretical ECCD profile. Comparison with the measured MSE data indicates the experimental data is consistent with the ECCD profile predicted by theory. The simulations yield a lower limit on the magnitude of the ECCD which is at or above the value found in Fokker-Planck calculations of the ECCD including quasilinear and parallel electric field effects.
\end{abstract}

\section{Introduction}

Current drive by electron cyclotron waves (ECCD) has been clearly observed in the DIII-D tokamak from near the magnetic axis out to half of the minor radius at a variety of poloidal locations [1,2]. The details of the system for generating and launching the electron cyclotron waves are discussed elsewhere [3]. For the purpose of this paper, it is only important to note that about $1 \mathrm{MW}$ of power is injected into the plasma at $110 \mathrm{GHz}$ which corresponds to the second harmonic of the electron cyclotron frequency. The waves are launched in a Gaussian beam which can be modeled in the far field as a point source at the final launching mirror with a divergence characterized by a 1.7 degree half width at half maximum. This model is consistent with in-vessel measurements of the antenna pattern in air. The current drive inferred in the analysis appears at a location consistent with the intersection of the launched beam with the second harmonic resonance in the plasma when the effects of refraction are included.

\section{Experimental Analysis}

The ECCD is inferred from the temporal and spatial evolution of the poloidal flux $\psi$ obtained from magnetic equilibrium reconstructions [4]. The requisite accuracy in the reconstruction is only obtained when the internal magnetic pitch angle measurements from the motional Stark effect (MSE) diagnostics are included. For any discharge, the total parallel current density $\mathrm{J}_{\|}$is given by spatial derivatives of $\psi$, while the Ohmic current density $\mathrm{J}_{\mathrm{OH}}$ is given by $\sigma_{\text {neo }} \mathrm{E}_{\|}$, where $\sigma_{\text {neo }}$ is the neoclassical conductivity and $E_{\mu}$ is the parallel electric field determined by a time derivative of $\psi$. The validity of using the neoclassical conductivity was demonstrated in Ref. [4] and is assumed to hold exactly in the absence of magnetohydrodynamic (MHD) instabilities such as sawteeth and tearing modes. Subtracting the Ohmic from the total current density gives the non-inductive current density $\mathrm{J}_{\mathrm{NI}}$. This $\mathrm{J}_{\mathrm{NI}}$ includes the bootstrap current and neutral beam current drive (NBCD) as well as the ECCD. The ECCD is isolated by comparing two discharges with similar parameters with and without ECCD. The difference in $J_{\mathrm{NI}}$ between the two dis- 
charges is attributed to ECCD. A small correction for changes in the kinetic profiles or the neutral beam power is applied using the ratios of the predicted NBCD and bootstrap current. Note that this correction does not require the absolute values or the theoretical spatial profile of the NBCD or bootstrap current to be accurately modeled; only the parametric dependence need be correct.

The ECCD inferred using this technique is localized about the predicted location as shown in Fig. 1. In both cases, the location of the measured ECCD (shown with error bars) agrees with predicted location based on ray tracing with the cold plasma dispersion relation and wave absorption calculated using the bounced-averaged Fokker-Planck equation [5]. (The profiles for each of the steps described above leading to the ECCD profiles can be found in Ref. [1].) The error bars on the experimental analysis are the effect of random error in determine $E_{\|}$, propagated through the calculation. This is the dominant source of random error in the analysis. In both cases, the resolution of the ECCD profile does not appear to be limited by random error.

For ECCD both near the magnetic axis and near the half radius, the inferred ECCD profile is significantly broader than that predicted by theory. This is true of the entire ECCD dataset analyzed so far. Several potential explanations for this effect have been identified; namely, finite spatial resolution of the analysis technique (akin to the more familiar instrumental broadening of spectroscopic measurements), antenna pattern broadening of unknown origin, and anomalous current diffusion due to particle transport or MHD instability at an undetectable level. The objective of the work reported here was to assess the intrinsic spatial resolution of the analysis technique by calculating the response of the motional Stark effect (MSE) diagnostic [6] to the ideal case where the current profile evolved according to the neoclassical Ohm's law and the theoretical ECCD profile. By comparing to the actual MSE signals, it should be possible to determine whether the broadening of the analyzed ECCD profile is a direct result of the input data or arises in the analysis technique itself.

\section{Simulations}

The simulation of the current profile evolution is carried out using the measured spatial and temporal profiles of the electron density, carbon density, electron temperature, ion temperature, and toroidal rotation as boundary conditions. The magnetic equilibrium reconstruction at the time when the EC power is turned on is used as an initial condition for the current profile evolution, and the boundary shape is used at all times as a boundary condition. A 1-1/2 D transport code [7] is used to step forward the current profile using the neoclassical Ohm's law and new magnetic equilibria are generated at $10 \mathrm{~ms}$ time resolution, as in the original analysis. In simulations of discharges with ECCD, a ray tracing code calculated the ECCD profile from the cold plasma dispersion relation and a linear current drive calculation $[8,9]$. The profile of the ECCD from the
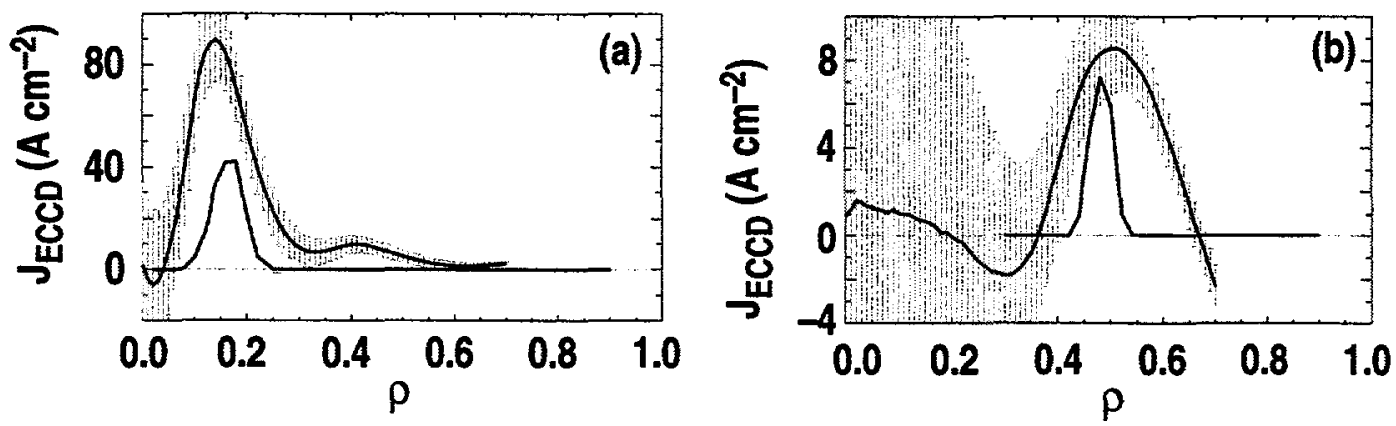

Fig. 1. The radial profiles for the case of (a) near central ECCD and (b) ECCD near the half radius. The discharge parameters for the central case are $B=1.76 \mathrm{~T}, \mathrm{I}=0.89 \mathrm{MA}, \mathrm{n}=1.7 \times 10^{13} \mathrm{~cm}^{-3}, \mathrm{P}_{\mathrm{NB}}=2.6 \mathrm{MW}, \mathrm{P}_{\mathrm{EC}}=1.1 \mathrm{MW}$. The discharge parameters for the half radius case are $B=1.86 \mathrm{~T}, \mathrm{I}=0.94 \mathrm{MA}, \mathrm{n}=1.8 \times 10^{13} \mathrm{~cm}^{-3}, \mathrm{P}_{\mathrm{NB}}=2.6 \mathrm{MW}$, $P_{E C}=1.0 \mathrm{MW}$. The curves without error bars are Fokker-Planck calculations of the ECCD including the effects of $E_{\text {H. }}$. In the near-central case the Fokker-Planck result has been scaled by 0.25 for comparison. 
Fokker-Planck calculation [5] is not significantly different in shape from the one used. To model the effects of quasilinear modification of the distribution function and synergy with the parallel electric field seen in the Fokker-Planck calculation, it is sufficient to raise the input power in the linear model. Given the poloidal flux from the simulated equilibria and the geometry of the DIII-D MSE system [6], it is straightforward to generate time histories of simulated MSE signals. Up to now, the finite spatial resolution of the MSE system has been ignored.

It is not possible to directly compare the simulated MSE pitch angles with the measured pitch angles because the spatial profile of the calculated NBCD does not match the experimentally measured profile. A useful quantity for comparing the experiment with the simulation is the change in pitch angle between two adjacent channels as can be seen by the following qualitative argument. The MSE system measures the pitch angle $\gamma$ of the total electric field. For the DIII-D system, to a good approximation, $\tan \gamma \propto \mathrm{B}_{z} / \mathrm{B} \phi$. From Amp ere's law in cylindrical coordinates, $J_{\phi} \simeq-1 / \mu_{0}\left(\partial B_{Z} / \partial R\right)$, neglecting the $\partial B_{R} / \partial z$ term since the measurement is very near the midplane. For small $\gamma, \tan \gamma \simeq \gamma$ and assuming $\mathrm{J}_{\phi} \approx \mathrm{J}_{\|}$, then the change in pitch angle between two adjacent channels $\Delta \gamma$ is proportional to the change in parallel current between the channels $\Delta \mathrm{J}_{\|}$. As in the experimental analysis, the effect of the ECCD can be isolated by taking the difference between $\Delta \gamma$ in discharges with and without ECCD. This quantity $\delta \Delta \gamma$ is proportional to $\mathrm{J}_{\mathrm{ECCD}}$ and the Ohmic current density generated by the back emf associated with the driven current. Although $\delta \Delta \gamma$ is the quantity most directly comparable with the MSE data, the quantity $\delta \Delta \mathrm{B}_{\mathrm{Z}}$ will be plotted to allow comparison of different views of the MSE system.

The width of the simulated MSE response to the ECCD agrees quite well with the experimental data, as shown in Fig. 2. The simulation used the experimental profiles and the launched beam parameters of the off-axis ECCD case shown in Fig. 1(b). Figure 2 shows good agreement of the simulations with both the tangential and edge MSE array data. The direct evidence of the current is the positive increase in $\mathrm{B}_{\mathrm{Z}}$ at the predicted location of the ECCD as shown in Fig. 2. The negative response to the small $\rho$ side of the ECCD is a result of the local back emf response to the ECCD diffusing toward a flat $E_{\|}$profile at a reduced level. The structure to the larger $\rho$ side of the ECCD in the targential channels does not appear in all cases. The conclusion is that the experimental MSE response is consistent with the ECCD profile which is predicted on the basis of the expected illumination of the resonance, given ray tracing of the expected beam.
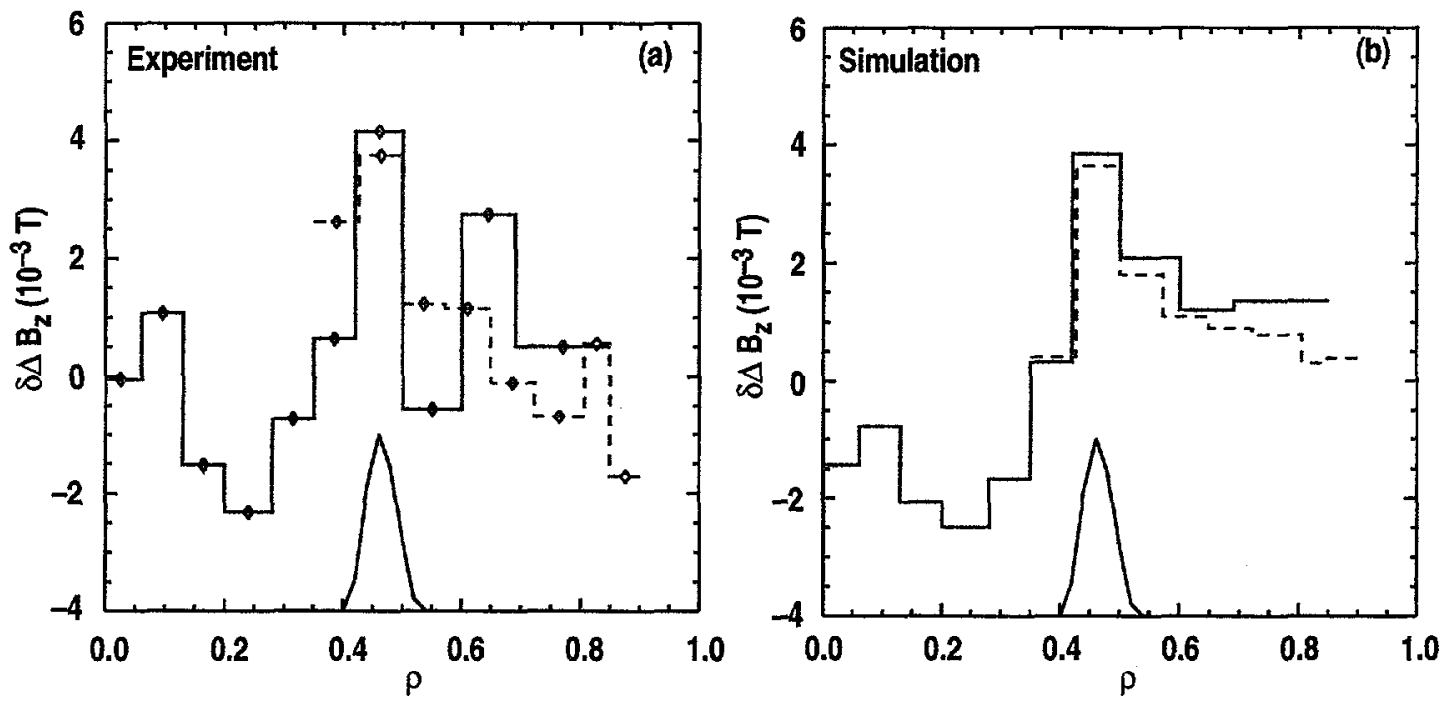

Fig. 2. (a) The difference between the change in $B_{z}$ from channel-to-channel of the MSE signals for the ECCD and NB-only fiducial. (See text for complete description.) The two curves correspond to two separate views from the MSE system on DIII-D. The solid curves are the tangential view and the dashed curve is the edge view. (b) The same quantity generated from the simulations for both of the arrays. The MSE signals are computed ignoring spatial averaging and finite beam stopping effects. The solid curve at the bottom of each figure is the theoretical ECCD profile. 
A second test of consistency between the time-dependent equilibrium analysis and the MSE data is to input the inferred experimental ECCD profile into the simulation and compare the simulated MSE signals with the experimental data. An example of this test is shown in Fig. 3. Two distinct qualitative differences appear between the simulation and the original data. The location of the peak forward response is shifted outward and is broader in the simulation compared to the experimental data. (The systematic structure in the experimental data weakens this conclusion somewhat for this case. However, this conclusion is borne out in all cases simulated so far.) Also, back emf response on the smaller $\rho$ side has diffused to the magnetic axis and is noticably larger. It appears that this broader profile is not consistent with the input data.

From the simulations presented in Figs. 2 and 3, the conclusion can be drawn that the MSE data are consistent with the narrow ECCD profile predicted by theory, while the ECCD profile inferred from the time histories of the magnetic equilibrium reconstructions is broader due to finite resolution of the technique. The latter conclusion is perhaps not too surprising, given that the magnetic reconstruction began with rather smooth basis functions for $\mathrm{ff}^{\prime}$ and $\mathrm{p}^{\prime}$ as the basis for the solution to the Grad-Shafranov equation [10]. (The function $\mathrm{f}$ is $\mathrm{RB}_{\phi} / \mu_{0}$ and $\mathrm{p}$ is the total plasma pressure.) A reduced $\chi^{2}$ test was used to determine the maximum number of basis functions to include in the reconstructions for each discharge. The basis functions in this case are cubic splines and to minimize bias an automatic routine was written to find the optimum knot locations for each discharge. The simulations show that all of the local structure of the ECCD appears in $\mathrm{ff}^{\prime}$ since the smooth fitted $\mathrm{p}^{\prime}$ from the experimental data is used. The spline functions are found to be surprisingly inefficient at reproducing the local structure in $\mathrm{ff}^{\prime}$ from the ECCD simulations; more knots are required than the reduced $\chi^{2}$ test would allow in the experimental case. This points out a potential improvement in the experimental analysis if an efficient set of basis functions could be found and implemented in the equilibrium reconstruction code.

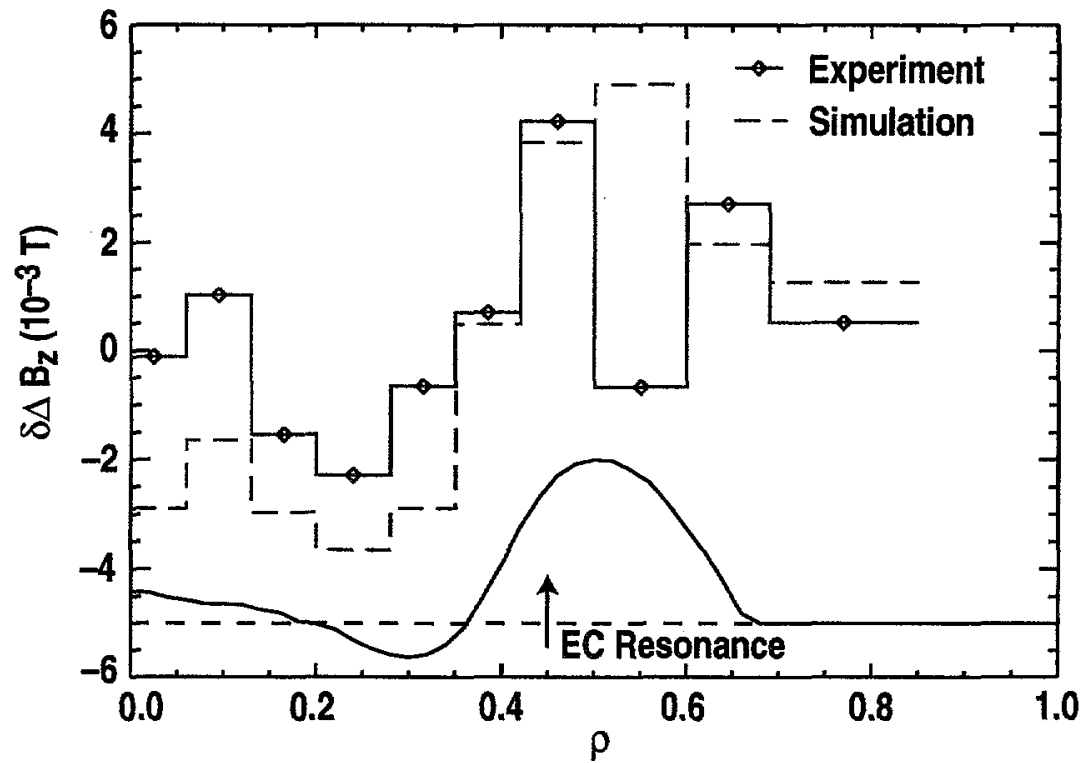

Fig. 3. Comparison of measured $\delta \Delta \mathrm{B}_{\mathrm{z}}$ for the tangential array (solid line) and the calculated value (dashed line) using the ECCD profile inferred from the time history of the magnetic equilibrium reconstruction. The inferred ECCD profile is shown at the bottom along with an indication of the resonance location.

The simulations also provide a test of the magnitude of the ECCD. Figure 4 shows comparison of simulated current drive at a level consistent with the Fokker-Planck predictions and also at the level indicated by the experimental analysis. The data is best described by a magnitude of current drive between the Fokker-Planck prediction and the value determined from 


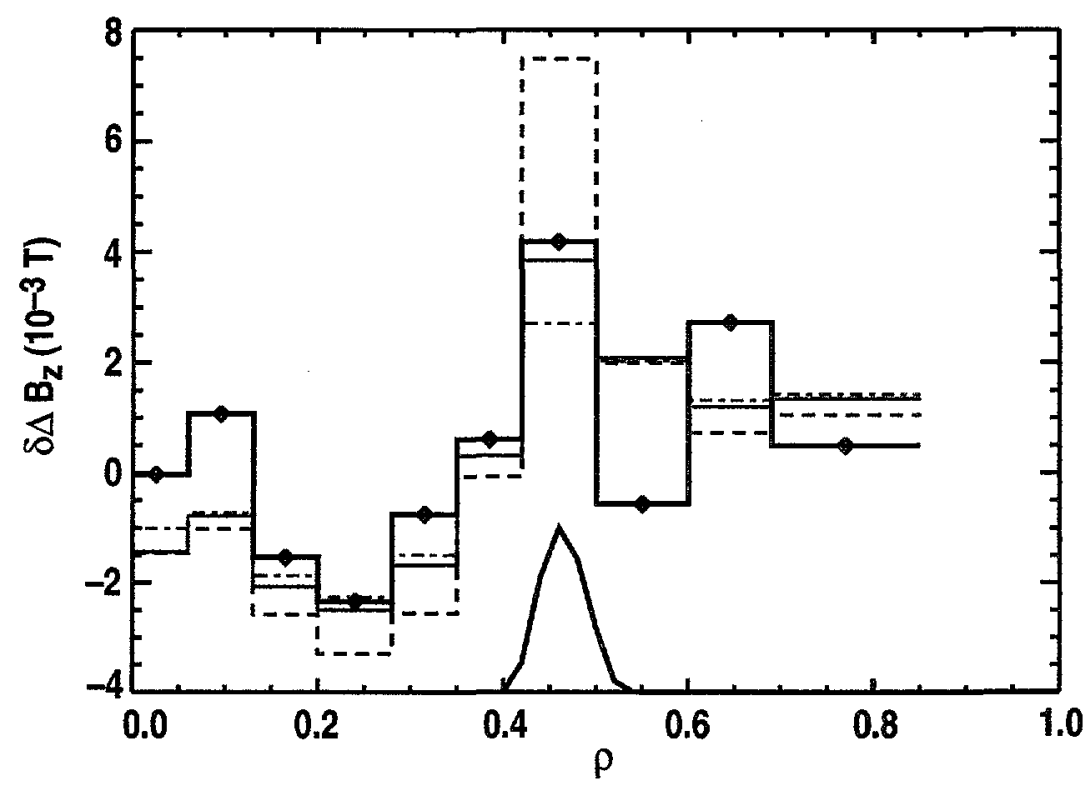

Fig. 4. Effect on $\delta \Delta \mathrm{B}_{\mathrm{z}}$ of changing the magnitude of the ECCD. The experimental data are shown with a solid line and a diamond symbol. Shown are simulations with the Fokker-Planck, experimentally inferred values, and the best matched case. The $\delta \Delta \mathrm{B}_{\mathrm{z}}$ for the forward current point is best matched for a magnitude of ECCD of $15 \mathrm{kA}$ (solid line) compared to $5 \mathrm{kA}$ for the linear case (not shown), $8 \mathrm{kA}$ for the Fokker-Planck calculation (chain-dot line), and $35 \mathrm{kA}$ for the experimental analysis (dashed line).

the experimental analysis. There are some indications that the simulation is not completely describing the experimental situation. No single value of current drive can describe both the forward response and the back emf response. Also, the diffusion of the back emf to the magnetic axis is faster in the simulation than indicated in the experiment (not shown). This suggests that the effective conductivity is actually higher in the experimental case than in the Ohm's law used in the simulation. A higher conductivity in the experiment is consistent with both the finite collisionality effects discussed in Ref. [1] and with a modified conductivity due to quasilinear distribution effects as seen in the Fokker-Planck calculation. The particular discharge simulated in this paper has a substantial enhancement of the predicted ECCD due to the finite $\mathrm{E}_{\mid}$. A higher conductivity would lessen the forward response at a given time and would yield a more localized and therefore larger back emf response to the ECCD. Therefore, the use of the forward response to gauge the magnitude of the ECCD provides a lower limit. Tests of this effect in the simulation will be carried out in the near future. At this point, the lower limit for the magnitude of the ECCD inferred from the simulations is at or above the Fokker-Planck prediction in all cases tested.

\section{Conclusions}

The simulations show very clearly that the experimental MSE data are consistent with a localized ECCD source of the shape predicted by theory. This significantly reduces the possibility of some anomalous current transport or broadening of the launched Gaussian beam and provides validation for proposed applications for ECCD which require highly localized current drive such as stabilization of MHD modes. The broadening which was apparent in the experimental analysis $[1,2]$ appears to be the result of finite spatial resolution of the present equilibrium reconstruction technique. It is still not possible to draw a strong conclusion from the simulations about the magnitude of the driven current. For off-axis ECCD cases, the simulations indicate magnitudes at or above the Fokker-Planck predictions. It is hoped that further refinement of the simulations and the experimental analysis will bring the two approaches into agreement. 
The planned increase in EC power to the plasma to the $3 \mathrm{MW}$ level in the coming year should also help clarify the magnitude of the ECCD.

\section{Acknowledgments}

This is a report of work supported by the U.S. Department of Energy under Contract Nos. DE-AC03-99ER54463 and W-7405-ENG-48. It is a pleasure to acknowledge helpful discussions with H.E. St. John, B.W. Rice, and Y.R. Lin-Liu.

\section{References}

[1] T.C. Luce, et al., "Generation of Localized Non-Inductive Current by Electron Cyclotron Waves on the DIII-D Tokamak," to be published in Phys. Rev. Lett., and General Atomics Report GA-A23018, May 1999.

[2] T.C. Luce, et al., "Modification of the Current Profile in DIII-D by Off-Axis Electron Cyclotron Current Drive," to be published in Plasma Phys. and Contr. Fusion, and General Atomics Report GA-A23167, July 1999.

[3] J.M. Lohr, et al., "The $110 \mathrm{GHz}$ Gyrotron System on DIII-D: Gyrotron Tests and Results," in the Proc. of the 4th Int. Workshop on Strong Microwaves in Plasmas, Nizhny Novgorod, Russia, 1999, to be published.

[4] C.B. Forest, et al., Phys. Rev. Lett. 73, 2244 (1994).

[5] R.W. Harvey, M.C. McCoy, in Advances in Simulation and Modeling of Thermonuclear Plasmas (Proc. of the IAEA Tech. Com. Mtg, Montreal, 1992) (International Atomic Energy Agency, Vienna, 1993) p. 498.

[6] B.W. Rice, et al., Phys. Rev. Lett. 79, 2394 (1997).

[7] H.E. St. John, et al., Plasma Phys. and Contr. Nucl. Fusion Research 1994 (Proc. of the 15th IAEA Conf., Seville, 1994) Vol. 3 (International Atomic Energy Agency, Vienna, 1994) p. 603.

[8] K. Matsuda, IEEE Trans. Plasma Sci. 17, 6 (1989).

[9] R.H. Cohen, Phys. Fluids 30, 2442 (1987).

[10] L.L. Lao, et al., Nucl. Fusion 30, 1035 (1990). 